\title{
Twinning Evolution as a Function of Loading Direction in Magnesium
}

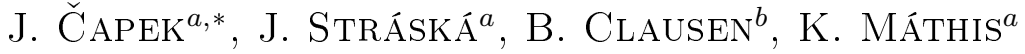 \\ ${ }^{a}$ Department of Physics of Materials, Faculty of Mathematics and Physics, Charles University, \\ Ke Karlovu 5, 12116 Prague, Czech Republic \\ ${ }^{b}$ Los Alamos National Laboratory, Lujan Neutron Scattering Center, Los Alamos, NM 87545, USA
}

\begin{abstract}
The twinning activity in random textured cast magnesium during monotonic, room temperature tension and compression tests was monitored by neutron diffraction. Decrease of integrated intensity which characterizes the twinned volume fraction of selected reflections was compared to its Schmid factor. The comparison shows that twinned fraction correlates with the maximum value of the Schmid factor with high precision during tensile test and with the average value of the Schmid factor during compression test.
\end{abstract}

DOI: 10.12693 /APhysPolA.128.762

PACS: 81.05.Bx, 61.05.fm, 61.72.Mm

\section{Introduction}

Having the hexagonal close packed (hcp) structure and $c / a$ ratio close to the ideal value the deformation behavior of magnesium alloys differ from the others, e.g. fcc or bcc metals. At room temperature the plastic deformation usually cannot take place by dislocation slip only $[1,2]$. Thus, another deformation mechanism has to be activated. As it was shown by numerous authors [3], deformation twinning plays a key role in plastic deformation of magnesium. The most easily activated twinning mode is $\{10 \overline{1} 2\}\langle 10 \overline{1} 1\rangle$ extension twinning, which causes reorientation of the crystal lattice about $86.3^{\circ}$ and extension of the grain along its $c$-axis.

Unlike dislocation slip twinning exhibits a polar behavior. Different grains undergo twinning during tensile and compressive deformation and the evolution of the deformation texture depends on the sense of straining. There is an intensive discussion in the current literature [4-7] whether the activation of a given twin variant depends on its Schmid factor (SF), defined as $m=\cos \phi \cos \lambda$, where $\phi$ and $\lambda$ are the angles between the loading direction and the twinning plane normal and twinning direction, respectively.

The neutron diffraction (ND) method has been successfully applied for the study of twinning in magnesium [8]. The main advantage of the ND method is in its capability to examine large sample volume, due to the deep penetration length of thermal neutrons. The information about twinning can be acquired by following the intensity changes of particular diffraction peaks [810]. In the present paper the Schmid factor analysis is combined with the in situ ND study of the twinning evolution in a randomly textured $\mathrm{Mg}-\mathrm{Al}$ magnesium alloy. Correspondence between the experimentally established

${ }^{*}$ corresponding author; e-mail: jan.capek@centrum.cz twinned volume fraction and the calculated Schmid factors for extension twinning (TwSF) in particular grain families is analyzed. ${ }^{\dagger}$

\section{Material and experimental methods}

Binary $\mathrm{Mg}-2$ wt\% Al alloy was used for the experiment. The ND measurement showed that the initial samples have random grain orientation distribution (Fig. 1). The monotonic compression and tensile tests were performed at room temperature at a strain rate of $10^{-3} \mathrm{~s}^{-1}$. The test was stopped for approximately $70 \mathrm{~min}$ at particular strain values $(0.1 \%, 0.5 \%, 1 \%, 2 \%, 3 \%, 4 \%, 5 \%$, $6 \%$ ) in order to collect the neutron diffraction data. The SMARTS engineering instrument at LANSCE was used for collecting the diffraction pattern [11]. The diffraction patterns were measured using two detector banks at $\pm 90^{\circ}$ to incident beam. The angle between the incident beam and the loading direction was $45^{\circ}$. This setup provides diffraction measurement of crystallographic planes both perpendicular (axial detector) and parallel (transversal detector) to the loading direction. We evaluated data from the axial detector which shows the grain orientation with respect to the loading direction.

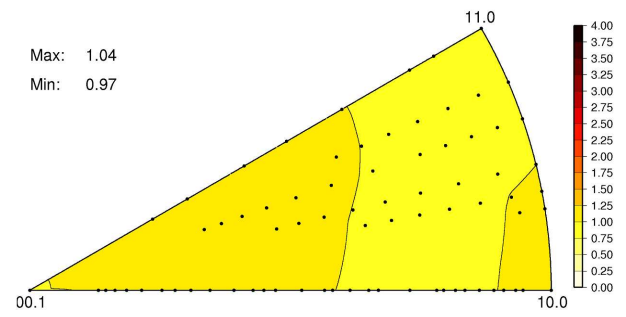

Fig. 1. Inverse pole figure of the compression sample in axial (loading) direction.

\footnotetext{
${ }^{\dagger}$ Contraction $\{10 \overline{1} 1\}$ twins were not considered due to the low strain levels achieved.
} 
Direct observations of the microstructure of the specimen deformed to $1 \%$ strain were performed by scanning electron microscope FEI Quanta 200F. The electron backscatter diffraction (EBSD) was used to study of the activated twin variants. The specimens for EBSD investigations were polished by standard methods down to OPS colloidal silica suspension. The final electropolishing was done by means of Struers AC-2-II electrolyte.

\section{Results and discussion}

Orientation dependence of the calculated TwSF is depicted in Fig. 2. The measured orientations, representing grain families with a particular orientation are highlighted. ${ }^{\ddagger}$ As it was shown in our concurrent paper [12], there is a significant difference between the compression and tension case. In compression, if the $c$-axis of a particular grain is deviated from the ideal orientation (i.e. when the $c$-axis is perpendicular to the loading direction) by more than $47^{\circ}$, the TwSF for all twin variants is $\leq 0$. On the contrary, in tension the $c$-axis deviation angle (the ideal orientation for tension is $c$-axis parallel to loading direction) is larger $\left(\approx 65^{\circ}\right)$ and for some grains exists at least one twin variant, which has very low $(<0.1)$, but non-negative zero Schmid factor above this limit.

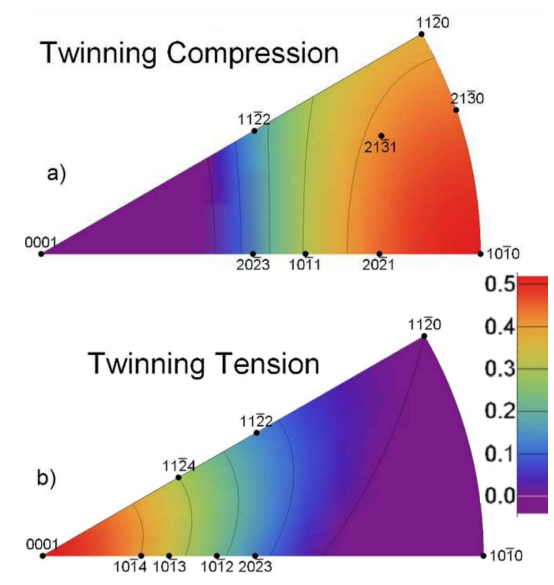

Fig. 2. Orientation dependence of Schmid factor for extension twinning (a) in compression, (b) in tension.

The TwSF for all six variants in the measured grain families and their deviation of their $c$-axes from the ideal orientation are listed in the Table. A negative value means that the particular twin variant causes deformation against the sense of loading direction.

There is a distinct difference between the tension and compression values. Close to the ideal orientation at least four twin variants have similar, large TwSF in tension, whereas only two in compression.

$\ddagger$ Only reflections with decreasing intensities (i.e. grains favorably oriented for twinning) were taken into account. Therefore, the grain families considered in tension and compression respectively are different.
TABLE

Deviation of $c$-axes and Schmid factors of selected orientations for all 6 variants of extension twinning, maximum and average value are highlighted.

\begin{tabular}{c|c|c|c|c|c|c|c}
\hline \hline \multicolumn{7}{c}{ during compression } \\
\hline Reflection & $\mathbf{1 0 . 0}$ & $\mathbf{2 0 . 1}$ & $\mathbf{2 1 . 0}$ & $\mathbf{2 1 . 1}$ & $\mathbf{1 1 . 0}$ & $\mathbf{1 0 . 1}$ & $\mathbf{1 1 . 2}$ \\
\hline Deviation & $0^{\circ}$ & $15^{\circ}$ & $0^{\circ}$ & $11^{\circ}$ & $0^{\circ}$ & $28^{\circ}$ & $31^{\circ}$ \\
\hline Variant 1 & 0.50 & 0.45 & 0.45 & 0.42 & 0.37 & 0.31 & 0.16 \\
Variant 2 & 0.12 & 0.09 & 0.02 & 0.00 & 0.00 & 0.00 & -0.14 \\
Variant 3 & 0.12 & 0.08 & 0.29 & 0.25 & 0.37 & -0.03 & 0.11 \\
Variant 4 & 0.50 & 0.42 & 0.45 & 0.40 & 0.37 & 0.25 & 0.11 \\
Variant 5 & 0.12 & 0.08 & 0.02 & 0.00 & 0.00 & -0.03 & -0.14 \\
Variant 6 & 0.12 & 0.09 & 0.29 & 0.26 & 0.37 & 0.00 & 0.16 \\
\hline max & $\mathbf{0 . 5 0}$ & $\mathbf{0 . 4 5}$ & $\mathbf{0 . 4 5}$ & $\mathbf{0 . 4 2}$ & $\mathbf{0 . 3 7}$ & $\mathbf{0 . 3 1}$ & $\mathbf{0 . 1 6}$ \\
average & $\mathbf{0 . 2 5}$ & $\mathbf{0 . 2 0}$ & $\mathbf{0 . 2 5}$ & $\mathbf{0 . 2 2}$ & $\mathbf{0 . 2 5}$ & $\mathbf{0 . 0 9}$ & $\mathbf{0 . 0 5}$ \\
\hline \hline \multicolumn{7}{|c}{ during tension } \\
\hline Reflection & $\mathbf{0 0 . 2}$ & $\mathbf{1 0 . 4}$ & $\mathbf{1 0 . 3}$ & $\mathbf{1 1 . 4}$ & $\mathbf{1 0 . 2}$ & $\mathbf{1 1 . 2}$ & $\mathbf{2 0 . 3}$ \\
Deviation & $0^{\circ}$ & $25^{\circ}$ & $32^{\circ}$ & $39^{\circ}$ & $43^{\circ}$ & $59^{\circ}$ & $51^{\circ}$ \\
\hline Variant 1 & 0.50 & 0.40 & 0.34 & 0.30 & 0.22 & 0.14 & 0.14 \\
Variant 2 & 0.50 & 0.37 & 0.31 & 0.12 & 0.19 & -0.16 & -0.10 \\
Variant 3 & 0.50 & 0.29 & 0.19 & 0.12 & 0.00 & -0.16 & -0.13 \\
Variant 4 & 0.50 & 0.37 & 0.31 & 0.30 & 0.19 & 0.14 & 0.08 \\
Variant 5 & 0.50 & 0.40 & 0.34 & 0.18 & 0.22 & -0.11 & -0.13 \\
Variant 6 & 0.50 & 0.34 & 0.25 & 0.18 & 0.06 & -0.11 & -0.10 \\
\hline max & $\mathbf{0 . 5 0}$ & $\mathbf{0 . 4 0}$ & $\mathbf{0 . 3 4}$ & $\mathbf{0 . 3 0}$ & $\mathbf{0 . 2 2}$ & $\mathbf{0 . 1 4}$ & $\mathbf{0 . 1 4}$ \\
average & $\mathbf{0 . 5 0}$ & $\mathbf{0 . 3 6}$ & $\mathbf{0 . 2 9}$ & $\mathbf{0 . 2 0}$ & $\mathbf{0 . 1 5}$ & $\mathbf{- 0 . 0 5}$ & $\mathbf{- 0 . 0 4}$
\end{tabular}

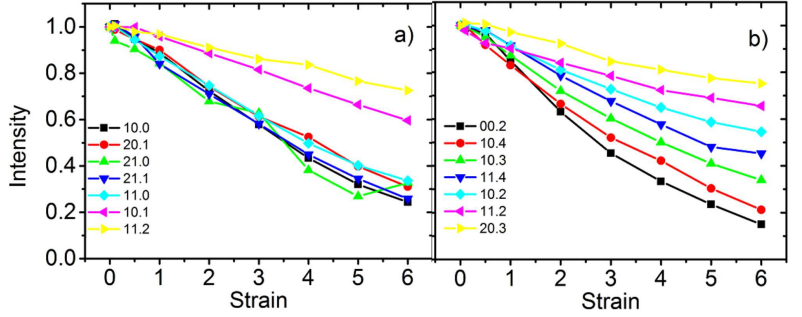

Fig. 3. Decrease of integrated intensities in favorable oriented grains (a) in compression, (b) in tension.

The change of the intensities of particular reflections with the applied strain is depicted in Fig. 3. The rate of the change is proportional to the twinned volume fraction, as it was first shown by Gharghouri et al. [8], i.e. a larger slope means more twinning.

Five reflections with the highest TwSF exhibit similar behavior in compression although the Schmid factors of their best twin variants vary a lot. It seems that the extension twinning is rather governed by the average value of the TwSF. This observation supports the conclusions of the works of Beyerlein et al. [5] and Guo et al. [7]. Based on the analysis of EBSD maps they found that in a compressed specimen the nucleation of a given twin variant strongly depends also on other parameters (grain boundary, orientation of the neighboring grains etc.). In particular cases, favorably oriented grains can remain untwinned or not the best twin variant is nucleated. Thus, the high maximum TwSF does not guarantee twin nucleation. Further, increase of number of twin variants 
per grain was found with decrease of maximum value of TwSF [5]. Such a behavior is presented on EBSD map in Fig. 4a. The TwSF of twin variants in the grain signed as $\mathrm{C} 1$ are $(0.44,0.23,0.01,0.41,0.21,0.01)$ and $(-0.06$, $0.25,0.25,-0.06,0.29,0.29)$ in the grain $\mathrm{C} 2$. It is obvious that all four allowed $(\mathrm{TwSF}>0)$ twin variants are present in grain $\mathrm{C} 2$. All these variants have similar TwSF. On the contrary, in the grain $\mathrm{C} 1$, where according to the theoretical calculations all six variants have a positive TwSF, only three variants were nucleated. The two with the highest TwSF are thick. The size of the third variant is small, despite of its TwSF value similar to that of twins in grain $\mathrm{C} 2$. This observation is in agreement with findings of Barnett [4], who pointed out that the nucleation ability and size of a particular variant depends on the ratio between the TwSF of the selected variant and the maximum TwSF for the considered grain orientation, respectively.

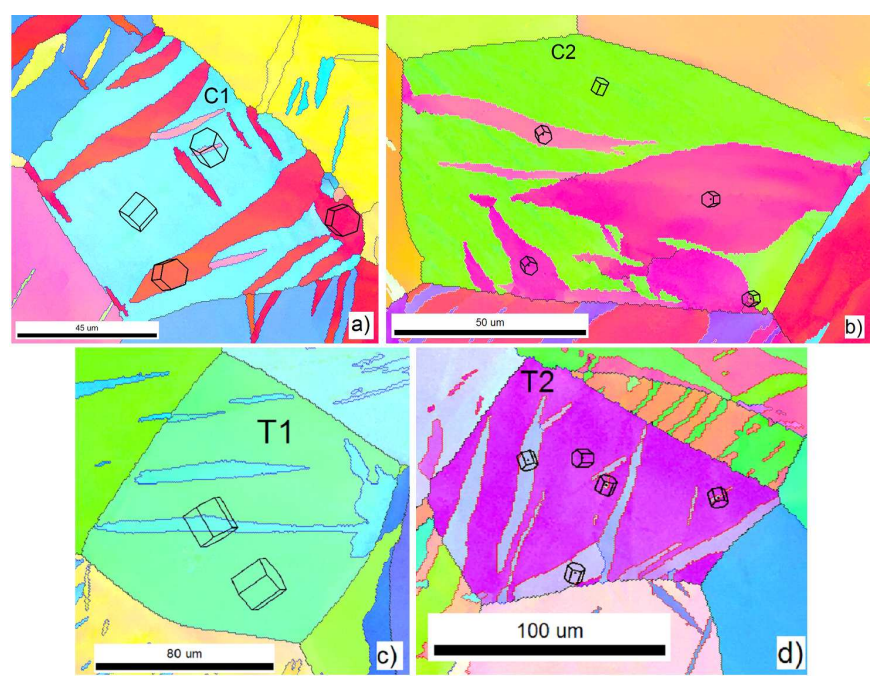

Fig. 4. EBSD maps of the microstructure after $1 \%$ strain $(\mathrm{a}, \mathrm{b})$ in compression, $(\mathrm{c}, \mathrm{d})$ in tension.

In tension, the rate of intensity drop corresponds to the values of TwSF. The largest drop (i.e. the larger twinned volume fraction) is observed for the best oriented grains and the twinning rate monotonically decreases with decreasing TwSF. In agreement with our previous work [12], we found twins also in unfavorable oriented grains, as it is presented in Fig. 4b. TwSF of grain T1 are $(0.01,-0.24$, $-0.38,0.02,-0.26,-0.40)$ and of grain $\mathrm{T} 2(0.08,0.11$, $-0.12,0.11,0.08,-0.18)$. In tension the number of nucleated twin variants per grain decreases with the decreasing value of maximum TwSF (see also Fig. 9a in [12]). Unlike in compression, the twinned volume growth cannot be realized with nucleation of additional twin variant. Thus, the twinned volume fraction in the grains with a particular orientation is a univocal function of their TwSF (independently, if we take the maximum or average value - nevertheless, due to the negative average values of $m$, considering the maximum value is physically more correct).

\section{Conclusion}

Extension $\{10 \overline{1} 2\}$ twinning was studied by comparing ND and EBSD measurements and the Schmid factor analysis. The following conclusions may be drawn:

Tension:

The twinning behavior of particular grains is consistent with their twinning Schmid factors. The largest twinned volume fraction was found for the best oriented grains and with decreasing Schmid factor the twin volume fraction decreased as well. Twins were found also in grains having very low $(<0.1)$ but positive Schmid factor.

Compression:

The variation of the twin volume fraction follows the average Schmid factor of all six variants rather than the best variant. A decrease of number of twin variants per grain was found with increase of value of the Schmid factor.

\section{Acknowledgments}

The authors are grateful for the financial support of the Czech Science Foundation under the contract 14-36566G. J.Č. acknowledges the support by the Charles University in Prague, Faculty of Mathematics and Physics, project GA UK No. 251715. This work has benefited from the use of the Lujan Neutron Scattering Center at LANSCE, funded by the US Department of Energy's Office of Basic Energy Sciences. Los Alamos National Laboratory is operated by Los Alamos National Security LLC under US DOE Contract DE-AC52-06NA25396.

\section{References}

[1] A. Chapuis, J.H. Driver, Acta Mater. 59, 1986 (2011).

[2] Magnesium and Magnesium Alloys (ASM Specialty Handbook), Eds. M.M. Avedesian, H. Baker, ASM International, Materials Park 1999.

[3] J.W. Christian, S. Mahajan, Prog. Mater. Sci. 39, 1 (1995).

[4] M.R. Barnett, Z. Keshavarz, A.G. Beer, X. Ma, Acta Mater. 56, 5 (2008).

[5] I.J. Beyerlein, L. Capolungo, P.E. Marshall, R.J. McCabe, C.N. Tome, Philos. Mag. 90, 2161 (2010).

[6] X.-L. Nan, H.-Y. Wang, L. Zhang, J.-B. Li, Q.C. Jiang, Scr. Mater. 67, 443 (2012).

[7] C. Guo, R. Xin, C. Ding, B. Song, Q. Liu, Mater. Sci. Eng. A 609, 92 (2014).

[8] M.A. Gharghouri, G.C. Weatherly, J.D. Embury, J. Root, Philos. Mag. A 79, 1671 (1999).

[9] S.R. Agnew, D.W. Brown, C.N. Tome, Acta Mater. 54, 4841 (2006).

[10] O. Muransky, D.G. Carr, P. Sittner, E.C. Oliver, Int. J. Plast. 25, 1107 (2009).

[11] M.A.M. Bourke, D.C. Dunand, E. Ustundag, Appl. Phys. A 74, S1707 (2002).

[12] J. Capek, K. Mathis, B. Clausen, J. Straska, P. Beran, P. Lukas, Mater. Sci. Eng. A 602, 25 (2014). 